\title{
A low-dimensional detector model for full counting statistics: Trajectories, Back-Action, and Fidelity
}

\author{
Gernot Schaller * Gerold Kießlich, and Tobias Brandes \\ Institut für Theoretische Physik, Hardenbergstraße 36, \\ Technische Universität Berlin, D-10623 Berlin, Germany
}

\begin{abstract}
We study the combined counting statistics of two capacitively coupled transport channels. In particular, we examine the conditions necessary for utilizing one channel as detector sensitive to the occupation of the other. A good detector fidelity may be achieved in a bistable regime when the tunneling rates through the two channels are vastly different - even when the physical back-action of the detector on the probed channel is large. Our methods allow to estimate the error of charge counting detectors from time-resolved current measurements - which have been obtained in recent experiments - alone.
\end{abstract}

Quantum transport is a generic example of nonequilibrium quantum dynamics 1 . A low-dimensional quantum system (e.g. quantum $\operatorname{dots}^{2}$, molecules ${ }^{\frac{3}{3}}$, nanotubes ${ }^{4}$ etc.) is typically coupled via particle exchange to multiple reservoirs held at different equilibria. The detection of stochastic particle transfer into a reservoir yields the full counting statistics (FCS) ${ }^{5}$. The FCS provides a tool to access system properties via an indirect measurement. The technique of $n$-resolved master equations 6 conveniently allows to extract the FCS from microscopic models (see e.g. $\stackrel{7}{\underline{7}}$ ). Recently, the value of this approach has been demonstrated experimentally by capacitively coupling a single quantum dot (QD) to a quantum point contact $(\mathrm{QPC})^{8}$. Here, we demonstrate that in principle, also two capacitively coupled QDs should show similar behavior as the QPC-QD configuration in certain parameter limits. However, the simplicity of our model enables us to estimate the physical detector back-action and the detection error from the FCS.

We consider a system composed of two nearby twoterminal single resonant level systems ${ }^{9} A$ and $B$ (compare Fig. 1a) $H_{\mathrm{S}}=\epsilon_{A} d_{A}^{\dagger} d_{A}+\epsilon_{B} d_{B}^{\dagger} d_{B}+U d_{A}^{\dagger} d_{A} d_{B}^{\dagger} d_{B}$, where $\epsilon_{A / B}$ denote the level energies of the single levels and $U$ models Coulomb interaction. The system is coupled to four fermionic reservoirs via $H_{\mathrm{SB}}=\sum_{k a} t_{k a, A} d_{A} c_{k a, A}^{\dagger}+\sum_{k a} t_{k a, B} d_{B} c_{k a, B}^{\dagger}+$ h.c., where $t_{k a, A / B}$ denote tunneling rates to the adjacent leads $a \in\{L, R\}$. We will use subsystem $A$ as a detector for the state of subsystem $B$. Transport through each level is enabled by applying nonvanishing bias voltages, and the two transport channels influence each other by the Coulomb interaction $U$.

Since we are interested in the charge FCS not only at one junction but at the interplay of the two channels, we introduce two virtual detectors in the tunneling terms associated with right leads (see also Ref $\underline{10}$ ). Following the Born-Markov-Secular ${ }^{11}$ approximation scheme, we arrive at an $\left(n_{A}, n_{B}\right)$-resolved master equation of the form

$$
\begin{aligned}
\dot{\rho}^{\left(n_{A} n_{B}\right)}= & \mathcal{L}_{00} \rho^{\left(n_{A} n_{B}\right)}+\mathcal{L}_{+0} \rho^{\left(n_{A}-1, n_{B}\right)}+\mathcal{L}_{-0} \rho^{\left(n_{A}+1, n_{B}\right)} \\
& +\mathcal{L}_{0+} \rho^{\left(n_{A}, n_{B}-1\right)}+\mathcal{L}_{0-} \rho^{\left(n_{A}, n_{B}+1\right)},
\end{aligned}
$$

where $\rho^{\left(n_{A} n_{B}\right)} \equiv\left\langle n_{A}, n_{B}|\rho| n_{A}, n_{B}\right\rangle$, which couples only the diagonals of the density matrix to each other. The total system (QDs and virtual detectors) density matrix can at all times be written as $\rho(t)=\sum_{n_{A}, n_{B}} \rho^{\left(n_{A} n_{B}\right)}(t) \otimes\left|n_{A}\right\rangle\left\langle n_{A}|\otimes| n_{B}\right\rangle\left\langle n_{B}\right|$, such that the probability to measure $n_{A}$ tunneled particles in the detector channel and $n_{B}$ tunneled particles in the system channel after time $t$ is given by $P_{n_{A} n_{B}}(t)=\operatorname{Tr}\left\{\rho^{\left(n_{A} n_{B}\right)}(t)\right\}$ (measurement postulate). Performing a two-dimensional Fourier-transform of Eq. (11) via $\rho(\chi, \xi, t) \equiv \sum_{n_{A}, n_{B}} \rho^{\left(n_{A} n_{B}\right)}(t) e^{i n_{A} \chi+i n_{B} \xi}$, we obtain a Fourier-transformed Liouvillian $\dot{\rho}(\chi, \xi, t)=\mathcal{L}(\chi, \xi) \rho(\chi, \xi, t)$ with two counting fields $\mathcal{L}(\chi, \xi) \equiv \mathcal{L}_{00}+e^{+i \chi} \mathcal{L}_{+0}+e^{-i \chi} \mathcal{L}_{-0}+e^{+i \xi} \mathcal{L}_{0+}+e^{-i \xi} \mathcal{L}_{0-}$. This equation can be solved formally, and for a given initial condition $\rho(0)=\rho_{0}^{(00)} \otimes|0\rangle\langle 0|\otimes| 0\rangle\langle 0|$, the density matrix $\rho^{\left(n_{A} n_{B}\right)}(\Delta t)$ can be obtained from the inverse Fourier transform. With the "superjump" superoperator

$$
\mathcal{J}^{\left(n_{A}, n_{B}\right)}(t) \equiv \frac{1}{4 \pi^{2}} \int_{-\pi}^{+\pi} e^{\mathcal{L}(\chi, \xi) t-i n_{A} \chi-i n_{B} \xi} d \chi d \xi
$$

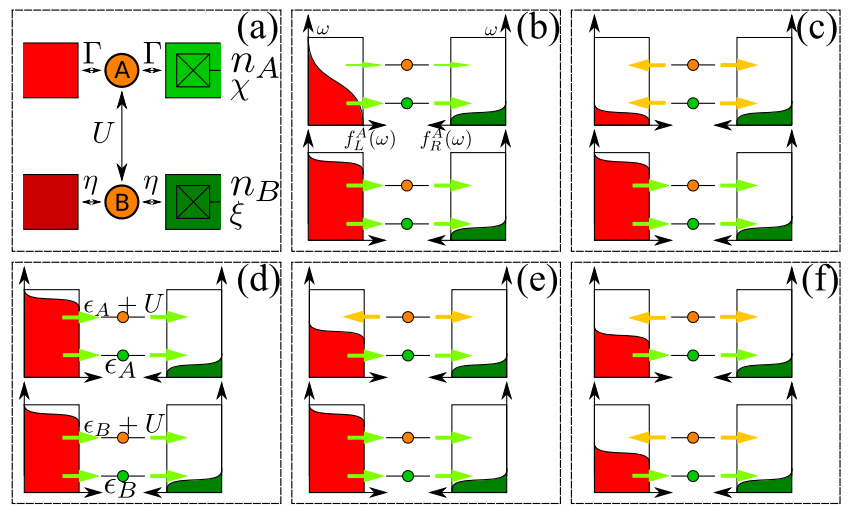

FIG. 1: (Color Online) (a) Spatial sketch of the physical setup. Tunneling is described by rates $\Gamma$ and $\eta$, respectively. Detectors are placed at the right junctions - with counting fields $\chi$ and $\xi$. Coulomb interaction $U$ leads to an effective shift of the dot levels (upper orange dots in panels b-f), which influences the current through each channel. (b-f) Bandscheme sketches with the associated Fermi functions $f_{L / R}^{A / B}(\omega)$ (labels in panel b) for different bias and temperature configurations (see text). 
we obtain after time $t$ for the density matrix $\rho^{\left(n_{A}, n_{B}\right)}(t)=\mathcal{J}^{\left(n_{A}, n_{B}\right)}(t) \rho_{0}^{(00)}$. Measuring the number of tunneled particles through both channels at this point will collapse the density matrix again. The explicit form of the Liouvillian considered in this work is given by a special bistable case of a size-scalable variant ${ }^{12}$ - with an additional counting field $\xi$.

We consider only the case of unidirectional transport as shown in Figs. 10-f: All right-associated Fermi functions vanish at the energy scales of the system $f_{R}^{j}\left(\epsilon_{j}\right)=f_{R}^{j}\left(\epsilon_{j}+U\right)=0$ (with $j \in\{A, B\}$ ), and the Liouvillian is given by $\mathcal{L}(\chi, \xi)=\Gamma \mathcal{L}_{A}(\chi)+\eta \mathcal{L}_{B}(\xi)$ with the flat tunneling rates $\Gamma \equiv 2 \pi \sum_{k}\left|t_{k a, A}\right|^{2} \delta\left(\omega-\omega_{k, a}\right)$ and $\eta \equiv 2 \pi \sum_{k}\left|t_{k a, B}\right|^{2} \delta\left(\omega-\omega_{k, a}\right)$. In the basis $\rho_{00,00}, \rho_{10,10}, \rho_{01,01}, \rho_{11,11}$, the superoperators read

$$
\begin{aligned}
\mathcal{L}_{A} & \equiv\left(\begin{array}{cc}
A\left(\epsilon_{A}\right) & 0 \\
0 & A\left(\bar{\epsilon}_{A}\right)
\end{array}\right), \mathcal{L}_{B} \equiv\left(\begin{array}{cc}
-B_{1}+B_{2}(\xi) \\
+B_{1} & -B_{2}(0)
\end{array}\right), \\
A(x) & \equiv\left(\begin{array}{cc}
-f_{L}^{A}(x) & e^{i \chi}+\bar{f}_{L}^{A}(x) \\
+f_{L}^{A}(x) & -1-\bar{f}_{L}^{A}(x)
\end{array}\right), \\
B_{1} & \equiv \operatorname{Diag}\left[\mathrm{f}_{\mathrm{L}}^{\mathrm{B}}\left(\epsilon_{\mathrm{B}}\right), \mathrm{f}_{\mathrm{L}}^{\mathrm{B}}\left(\bar{\epsilon}_{\mathrm{B}}\right],\right. \\
B_{2}(\xi) & \equiv \operatorname{Diag}\left[\mathrm{e}^{\mathrm{i} \xi}+\overline{\mathrm{f}}_{\mathrm{L}}^{\mathrm{B}}\left(\epsilon_{\mathrm{B}}\right), \mathrm{e}^{\mathrm{i} \xi}+\overline{\mathrm{f}}_{\mathrm{L}}^{\mathrm{B}}\left(\bar{\epsilon}_{\mathrm{B}}\right)\right],
\end{aligned}
$$

where $\bar{f}_{L / R}^{j}(x) \equiv 1-f_{L / R}^{j}(x)$ and $\bar{\epsilon}_{j} \equiv \epsilon_{j}+U$. Bistability occurs when e.g. $\eta \rightarrow 0$ and $f_{L}^{A}\left(\epsilon_{A}\right) \neq f_{L}^{A}\left(\bar{\epsilon}_{A}\right)$, since the block structure of the Liouvillian supports two different currents (and stationary states) in this case 12 .

Current Trajectories. When channel $B$ is at infinite bias and at sufficiently large temperatures $k_{\mathrm{B}} T \gtrsim U$, the left-associated Fermi functions $f_{L}^{A}\left(\epsilon_{A}\right)$ and $f_{L}^{A}\left(\bar{\epsilon}_{A}\right)$ will assume intermediate values between zero and one (see Fig 1b), which yields two different currents through A depending on whether $B$ is occupied or not. Ignoring the number of transferred charges through channel $B$, the probability of obtaining $n_{A}$ charges after time $\Delta t$ equates to $P_{n_{A}}(\Delta t)=\operatorname{Tr}\left\{\sum_{n_{B}} \mathcal{J}^{\left(n_{A}, n_{B}\right)}(\Delta t) \rho_{0}\right\} \equiv$ $\operatorname{Tr}\left\{\mathcal{J}_{A}^{\left(n_{A}\right)}(\Delta t) \rho_{0}\right\}$. That is, to obtain a trajectory of current measurements performed equidistantly at intervals $\Delta t$ numerically, a random number according to the distribution $P_{n_{A}}(\Delta t)$ must be drawn. The outcome of this then corresponds to a measurement of $n_{A}$ particles (with current $I_{A} \equiv n_{A} / \Delta t$ ). For simplicity, we exploit the translational invariance of Eq. (11) and shift $n_{A}$ to zero after each measurement. However, for the subsequent evolution, one now has to use the normalized density matrix $\rho^{\left(n_{A}\right)}$ as initial condition, which leads to a temporal correlation of the measured currents, see Fig. 2. In principle, also for unidirectional transport the number of different outcomes $\mathfrak{M} \in\{0,1,2, \ldots\}$ is infinite, but fortunately, there exists a natural cutoff as for $n_{A} \geq \Gamma \Delta t$ the operators $\mathcal{J}_{A}^{\left(n_{A}\right)}(\Delta t)$ become exponentially small. The trajectory in Fig. 2 is very similar to results obtained for QPCs 8 . The blips from low to high currents (arrows) should therefore indicate single charges tunneling from channel $B$ to its right junction. However, as tunneling through channel $A$ is also a stochastic process,

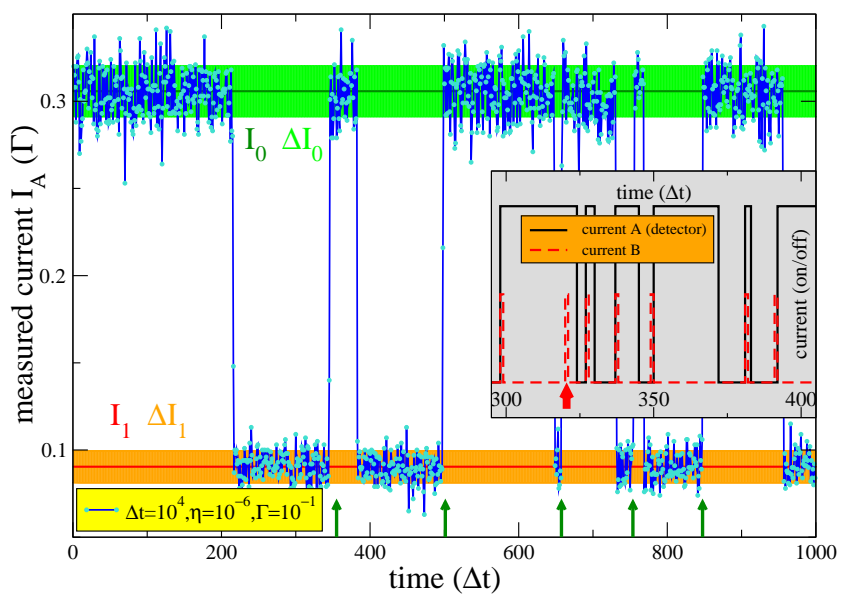

FIG. 2: (Color Online) Trajectory for configuration in Fig. 10 for 1000 current measurements at time intervals of $\Delta t$. Background lines display the currents and widths (shaded regions) for the single resonant level evaluated at the effective QD level one obtains for empty (high current) or filled (low current) channel $B$. Temperature and chemical potentials chosen such that $f_{L}\left(\epsilon_{A}\right)=0.612$ and $f_{L}\left(\bar{\epsilon}_{A}\right)=0.181$. Inset (parameters $\Gamma \Delta t=10, \eta \Delta t=0.1, \Delta t=100.0$ arb. u.) shows the correlation of both (coarse-grained) currents for the configuration in Fig. 11. When the detector current (solid black) rises, one most often also observes a spike (single charge) in the current through channel $B$ (dashed red, rescaled). However, due to stochastic behavior of the detector, jump events may be missed completely (bold red arrow).

jump events may in principle be missed (compare also the inset). Therefore, this raises the question of detector fidelity and back-action. In the following, we will investigate this for configurations (c)-(f) in Fig. 1.

Unperturbed Level (Fig. [1]). In this limit, the current through $A$ is blocked completely, such that the detector is turned off. With the replacements $f_{L}^{A}\left(\epsilon_{A}\right)=f_{L}^{A}\left(\bar{\epsilon}_{A}\right)=0$ and $f_{L}^{B}\left(\epsilon_{B}\right)=f_{L}^{B}\left(\bar{\epsilon}_{B}\right)=1$ in Eq. (3), we obtain for the Laplace transform $\tilde{M}(\chi, \xi, z) \equiv$ $\operatorname{Tr}\left\{[z \mathbf{1}-\mathcal{L}(\chi, \xi)]^{-1} \bar{\rho}\right\}$ of the moment-generating function (MGF)

$$
\tilde{M}(\chi, \xi, z)=\tilde{M}_{B}(\xi, z)=\frac{\left(3+e^{i \xi}\right) \eta+2 z}{2\left[z^{2}+2 \eta z-\left(e^{i \xi}-1\right) \eta^{2}\right]},
$$

from which one may analytically 12 deduce the probability distribution of a single resonant level in the symmetric infinite bias limit.

Infinite Bias (Fig. 1]d). When both channels are held at infinite bias - replacements $f_{L}^{A}\left(\epsilon_{A}\right)=f_{L}^{A}\left(\bar{\epsilon}_{A}\right)=1$ and $f_{L}^{B}\left(\epsilon_{B}\right)=f_{L}^{B}\left(\bar{\epsilon}_{B}\right)=1$ in Eq. (3) - the detector cannot distinguish the different states of the probed system. From the exact matrix exponential of the Liouvillian we obtain $P_{n_{A} n_{B}}(t)=P_{n_{A}}(t) P_{n_{B}}(t)$, where each probability distribution corresponds to that of a single resonant leve1 ${ }^{12}$ (with $\eta \rightarrow \Gamma$ for channel $A$ ). In Laplace space, this becomes visible by considering the reduction to Eq. (4) via $\tilde{M}(0, \xi, z)=\tilde{M}_{B}(\xi, z)$ and similarly 
$\tilde{M}(\chi, 0, z)=\tilde{M}_{A}(\chi, z)$. Clearly, we obtain for the first and second (long-term limit) cumulants of the detector (current and noise) $\left\langle\left\langle n_{A}\right\rangle\right\rangle=\frac{\Gamma t}{2}$ and $\left\langle\left\langle n_{A}^{2}\right\rangle\right\rangle \rightarrow \frac{1}{8}+\frac{\Gamma t}{4}$ and for the probed system $\left\langle\left\langle n_{B}\right\rangle\right\rangle=\frac{\eta t}{2}$ and $\left\langle\left\langle n_{B}^{2}\right\rangle\right\rangle \rightarrow \frac{1}{8}+\frac{\eta t}{4}$. From the factorization of the probability distributions it follows also that the cross-correlations vanish at all times $\left\langle\left\langle n_{A} n_{B}\right\rangle\right\rangle \equiv\left\langle n_{A} n_{B}\right\rangle-\left\langle n_{A}\right\rangle\left\langle n_{B}\right\rangle=0$.

Charge Detector Limit (Fig. 1 e). When $f_{L}^{A}\left(\epsilon_{A}\right)=$ $1, f_{L}^{A}\left(\bar{\epsilon}_{A}\right)=0$, and $f_{L}^{B}\left(\epsilon_{B}\right)=f_{L}^{B}\left(\bar{\epsilon}_{B}\right)=1$ in Eq. (3) - the detector is bistable $\frac{12}{2}$, which can be used to distinguish the two different states of channel $B$ : When channel $B$ is occupied, current through $A$ is blocked completely, whereas it is maximal (at its infinite bias value) otherwise. We find that the Laplace transform of the MGF fulfills $\tilde{M}(0, \xi, z)=\tilde{M}_{B}(\xi, z)$ - compare Eq. (4) - which demonstrates that here the physical action of the detector on the probed system vanishes. In contrast, the physical influence of the system on the detector is not negligible, which is demonstrated by $\tilde{M}(\chi, 0, z) \neq \tilde{M}_{A}(\chi, z)$. Therefore, the role of the detector will be purely informational (measurement postulate) without physical backaction on the system.

We obtain for the first cumulants $\left\langle\left\langle n_{A}\right\rangle\right\rangle=\frac{\Gamma t}{4}$, $\left\langle\left\langle n_{B}\right\rangle\right\rangle=\frac{\eta t}{2}$, whereas the second cumulants asymptotically approach the long-term limits

$$
\begin{aligned}
& \left\langle\left\langle n_{A}^{2}\right\rangle\right\rangle \rightarrow \frac{1}{32}\left(1-\frac{\Gamma^{2}}{\eta^{2}}\right)+\frac{3 \eta^{2}+3 \eta \Gamma+\Gamma^{2}}{16 \eta(\eta+\Gamma)} \Gamma t, \\
& \left\langle\left\langle n_{B}^{2}\right\rangle\right\rangle \rightarrow \frac{1}{8}+\frac{\eta}{4} t, \quad\left\langle\left\langle n_{A} n_{B}\right\rangle\right\rangle \rightarrow-\frac{\Gamma}{16(\eta+\Gamma)},(5)
\end{aligned}
$$

i.e., there exists a slight negative cross-correlation between the two channels, which surprisingly saturates.

In order to operate the channel $A$ as a detector for the FCS of channel $B$, we need to scan channel $A$ at much larger rates than $B(\Gamma \gg \eta)$. In particular, to obtain a meaningful detector current [associated with a transiently bimodal12 distribution $\left.P_{n_{A}}(\Delta t)\right]$, we require $\Gamma \Delta t \gg 1$ and to see the slow switching in time-resolved current measurements it is also necessary that $\eta \Delta t \ll 1$. Then, measuring a vanishing detector current $I_{A}$ within a time interval $\Delta t$ indicates with high probability that system $B$ is occupied, and measuring a non-vanishing detector current indicates an empty channel $B$.

To investigate within which limits on $\Gamma, \eta$, and $\Delta t$ this simplistic view is valid, we introduce the coarsegrained superoperators $\mathcal{J}^{L L} \equiv \mathcal{J}^{(00)}(\Delta t), \mathcal{J}^{L H} \equiv$ $\sum_{n_{B} \geq 1} \mathcal{J}^{\left(0 n_{B}\right)}(\Delta t), \mathcal{J}^{H L} \equiv \sum_{n_{A} \geq 1} \mathcal{J}^{\left(n_{A} 0\right)}(\Delta t)$, and $\mathcal{J}^{H H} \equiv \sum_{n_{A}, n_{B} \geq 1} \mathcal{J}^{\left(n_{A} n_{B}\right)}(\Delta t)$, corresponding to a vanishing $(L)$ or non-vanishing $(H)$ current through channels $A$ and $B$. That is, the $L / H$-discrimination threshold is set here independent of $\Delta t$. From Eq. (2), we may analytically obtain their Laplace transforms by performing the summation in frequency space (not shown). We may then create coarse-grained current trajectories with both detectors $A$ and $B$ (leading to four different measurement outcomes $\mathfrak{M} \in\{L L, L H, H L, H H\})$, see inset of Fig. 2 . Obviously, the correlation between blips of current $I_{A}$ and spikes of current $I_{B}$ is not perfect: The detector result may fail, as e. g. tunneling charges through channel $B$ may be missed.

Without the FCS of channel $B$, the error probability of the detector can be calculated from the averaged joint probabilities of measuring a large current in channel $A$ and a particle in channel $B$ and measuring a vanishing current through $A$ in combination with no particle in $B$ : $P_{\text {err }}^{A}(\Delta t) \equiv \operatorname{Tr}\left\{d_{B}^{\dagger} d_{B} \mathcal{J}_{A}^{H} \bar{\rho}\right\}+\operatorname{Tr}\left\{\left(\mathbf{1}-d_{B}^{\dagger} d_{B}\right) \mathcal{J}_{A}^{L} \bar{\rho}\right\}$, where $\bar{\rho}$ is defined via $\mathcal{L}(0,0) \bar{\rho}=0, \mathcal{J}_{A}^{H} \equiv \mathcal{J}^{H L}+\mathcal{J}^{H H}$, and $\mathcal{J}_{A}^{L} \equiv \mathcal{J}^{L L}+\mathcal{J}^{L H}$. Its Laplace transform equates to

$$
\tilde{P}_{\mathrm{err}}^{A}(z)=\frac{1}{2 z}-\frac{\Gamma^{2}\left[2(2 \Gamma+z)^{2}+\eta(7 \Gamma+4 z)\right]}{8(\Gamma+\eta) \mathcal{P}_{\text {num }}(z)}
$$

with $\mathcal{P}_{\text {num }}(z) \equiv 2 \eta \Gamma^{2}(\eta+\Gamma)+2 \Gamma(\eta+\Gamma)(4 \eta+\Gamma) z$ $+(2 \eta+\Gamma)(2 \eta+5 \Gamma) z^{2}+4(\eta+\Gamma) z^{3}+z^{4}$. From the Laplace transform we can directly conclude that for too short or too long measurement times the detector result will be useless: $\lim _{\Delta t \rightarrow 0} P_{\text {err }}^{A}(\Delta t)=\frac{1}{2}$ and $\lim _{\Delta t \rightarrow \infty} P_{\text {err }}^{A}(\Delta t)=$ $\frac{1}{2}$. An intuitive explanation This can be understood intuitively: For too short measurement times, the two peaks of the corresponding bimodal distribution will not have well separated, and measuring a vanishing particle number (current) $n_{A}(\Delta t)$ is still possible even when channel $B$ is empty. In contrast, for too large measurement times, we may obtain an average current over several cycles of loaded and empty channel $B$. For intermediate measurement times $\Delta t$ however, we observe a pronounced minimum of $P_{\mathrm{err}}^{A}(\Delta t)$, see Fig. 3 .

Dynamical Channel Blockade (Fig. 1ff). Here, both probed system $(B)$ and detector $(A)$ may be seen as bistable systems $\frac{12}{2}$, depending on whether $\eta$ or $\Gamma$ dominate, respectively. When occupied, both transport channels block the current through the other one completely $\underline{13}$. With the replacements $f_{L}^{A}\left(\epsilon_{A}\right)=1$, $f_{L}^{A}\left(\bar{\epsilon}_{A}\right)=0, f_{L}^{B}\left(\epsilon_{B}\right)=1$, and $f_{L}^{B}\left(\bar{\epsilon}_{B}\right)=0$ in Eq. (3), it becomes obvious that the doubly charged state $|11\rangle$ will not be occupied. When we choose the junctions to $A$ and $B$ and the corresponding tunneling rates identically $(\eta \rightarrow \Gamma$ and $\xi \rightarrow \chi)$, we recover the formal Liouvillian structure in Ref $\stackrel{14}{\underline{14}}$ for a two-terminal two-level system. The Laplace transform of the MGF $\tilde{M}(\chi, \xi, z)$ may also be obtained analytically, and we find that the interactions and back-actions between system and detector are not negligible, exemplified by $\tilde{M}(0, \xi, z) \neq \tilde{M}_{B}(\xi, z)$ and $\tilde{M}(\chi, 0, z) \neq \tilde{M}_{A}(\chi, z)$. From the MGF we obtain for the first cumulants $\left\langle\left\langle n_{A}\right\rangle\right\rangle=\frac{\Gamma t}{3},\left\langle\left\langle n_{B}\right\rangle\right\rangle=\frac{\eta t}{3}$, and the second cumulants approach the asymptotic limits

$$
\begin{aligned}
\left\langle\left\langle n_{A}^{2}\right\rangle\right\rangle & \rightarrow \frac{8 \eta^{2}-2 \eta \Gamma-4 \Gamma^{2}}{81 \eta^{2}}+\frac{5 \eta+2 \Gamma}{27 \eta} \Gamma t, \\
\left\langle\left\langle n_{B}^{2}\right\rangle\right\rangle & \rightarrow \frac{8 \Gamma^{2}-2 \eta \Gamma-4 \eta^{2}}{81 \Gamma^{2}}+\frac{2 \eta+5 \Gamma}{27 \Gamma} \eta t, \\
\left\langle\left\langle n_{A} n_{B}\right\rangle\right\rangle & \rightarrow+2 \frac{\eta^{2}-\eta \Gamma+\Gamma^{2}}{81 \eta \Gamma}-\frac{\eta+\Gamma}{27} t,
\end{aligned}
$$

such that the cross-correlations do now also appear in the currents. We may also here introduce the superoperators 


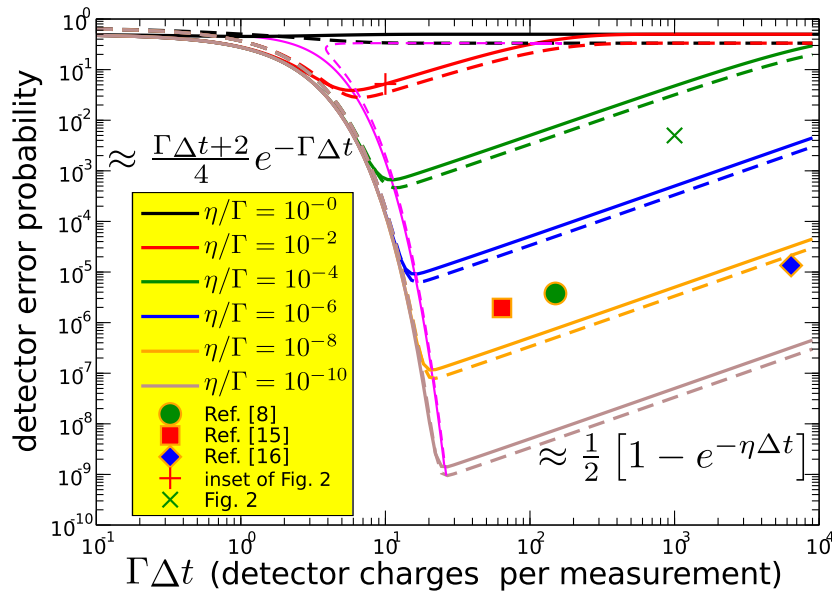

FIG. 3: (Color Online) Detector error probability in the quantum detector limit [Eq. (6), solid lines] and in the dynamical channel blockade limit [Eq. (8), dashed lines] for varying ratios of average tunneled detector and system charges $\eta / \Gamma$. For a too small or too large number of tunneled detector charges, the detector result is practically useless, whereas for an intermediate number, a pronounced minimum (note the logarithmic plot) of the error probability is found. Thin curves in the background mark the trajectory of the minimum. Asymptotic formula refer to solid lines (c) - dashed curve asymptotics would have slightly different prefactors. Filled symbols yield rough error estimates for experiments.

corresponding to either zero or more than zero particles leaving either transport channel, and the current trajectories (not shown) are similar to the inset of Fig. 2, The Laplace transform $P_{\mathrm{err}}^{A}(\Delta t)$ is obtained similarly

$$
\tilde{P}_{\text {err }}^{A}(z)=\frac{z^{2}(3 \Gamma+2 z)+\eta\left(\Gamma^{2}+6 \Gamma z+4 z^{2}\right)}{3 z(\Gamma+z)[z(\Gamma+z)+\eta(\Gamma+2 z)]},
$$

and we can directly conclude that for too short or too long measurement times the detector result will be useless since $\lim _{\Delta t \rightarrow 0} P_{\text {err }}^{A}(\Delta t)=\frac{2}{3}$ and $\lim _{\Delta t \rightarrow \infty} P_{\text {err }}^{A}(\Delta t)=\frac{1}{3}$. For finite measurement times the error probability will show a pronounced minimum, see Fig. 3. Therefore, measurements on $A$ may give reliable information on the state of $B$ (which differs strongly from its uncoupled dynamics)

Detector Errors and Experiments. Eqns. (6) and (8) generate a map of detector fidelity (Fig. 3). The detection error only depends on the two dimensionless variables $\Gamma \Delta t$ (number of detector charges during the measurement time) and $\eta / \Gamma$ (ratio of system to detector currents) and can be made extremely small. The striking similarity of the detector error probability for ideal and non-ideal detectors (also the similarity of the current trajectory in Fig. 2 with experiments for $\mathrm{QPCs}^{8}$ ) indicates that the simple dependence on detector and system charge throughput is generic for $I_{\mathrm{det}}^{\min } \ll I_{\mathrm{det}}^{\max }$. An error estimate taking into account a realistic $\Delta t$-scaling of the $I_{\text {low }} / I_{\text {high }}$ discrimination threshold, asymmetric and energy-dependent tunneling rates, multiple levels etc. would be much more accurate, but for a crude estimate we may link experimental parameters with those of our model: The width of the experimental current divided by its mean value will in the high-bias limit yield the average number of tunneled detector charges during the measurement time via $\Delta I_{\mathrm{det}}^{\max } / I_{\mathrm{det}}^{\max } \approx 1 / \sqrt{\Gamma \Delta t}$, and the ratio of mean system current (number of blips divided by time) and mean detector current yields $I_{\mathrm{sys}} / I_{\mathrm{det}}^{\max } \approx \eta / \Gamma$, and we obtain error probabilities of approximately $10^{-5}$ per measurement for Refs,$\frac{8,15,16}{}$.

Conclusions. Even with strong physical back-action the detector may yield reliable results for the instantaneous occupation of channel $B$ (with an altered dynamics). To decide whether the used detector strongly backacts on the probed system, it is therefore more useful to observe the scaling of the channel cross correlations: The smaller the scaling coefficient of the cross-cumulant in time, the smaller is the detector back-action. In order to estimate the fidelity of a single current measurement from Fig. 3, we propose to use the characteristics of timeresolved experimental detector current trajectories.

Financial support by the DFG (project BR 1528/5-1) and discussions with R. Sanchez are acknowledged.
* Electronic address: gernot.schaller@tu-berlin.de

1 Y. V. Nazarov and Y. M. Blanter, eds., Quantum Transport: Introduction to Nanoscience (Cambridge University Press, Cambridge, 2009).

2 L. P. Kouwenhoven et al., in Mesoscopic Electron Transport, (Kluwer Academic Publishers, Dordrecht, 1997).

3 J. Koch and F. von Oppen, Phys. Rev. Lett. 94, 206804 (2005).

4 S. Reich, C. Thomsen, and J. Maultzsch, Carbon Nanotubes: Basic Concepts and Physical Principles (WileyVCH, Weinheim, 2004).

${ }^{5}$ L. S. Levitov and G. B. Lesovik, JETP Lett. 58, 230 (1993).

6 S. A. Gurvitz and Y. S. Prager, Phys. Rev. B 53, 15932 (1996).

7 T. Brandes, Phys. Rep. 408, 315 (2005).
8 S. Gustavsson et al., Phys. Rev. Lett. 96, 076605 (2006).

${ }^{9}$ R. Sanchez et al., Phys. Rev. Lett. 104, 076801 (2010).

10 C. B. Doiron, B. Trauzettel, and C. Bruder, Phys. Rev. B 76, 195312 (2007).

11 G. Schaller and T. Brandes, Phys. Rev. A 78, 022106 (2008).

12 G. Schaller, G. Kießlich, and T. Brandes, Phys. Rev. B 81, 205305 (2010).

13 A. Cottet et al. Phys. Rev. B 70, 115315 (2004).

14 W. Belzig, Phys. Rev. B 71, 161301(R) (2005).

15 C. Fricke et al., Phys. Rev. B 76, 155307 (2007); C. Fricke, PhD thesis (2009), URL http://d-nb.info/995931283/34

16 E. V. Sukhorukov et al., Nature Phys. 3, 243 (2007). 